\title{
“Campo" en la educación médica: un escenario para poner en perspectiva prácticas de Salud y Educación
}

\author{
Gabriela Lucía Lacarta ${ }^{(a)}$ \\ Diana Milstein ${ }^{(\mathrm{b})}$ \\ Tomás Schwarcz ${ }^{(\mathrm{c})}$ \\ María Andrea Dakessian ${ }^{\text {(d) }}$ \\ Alicia Cattani(e)
}

\section{Introducción}

Este trabajo aborda situaciones que acontecen en el escenario de aprendizaje "Campo" del primer año de la carrera de medicina del Departamento de Ciencias de la Salud de la Universidad Nacional de La Matanza, Provincia de Buenos Aires, Argentina. Surge de la investigación que se está llevando a cabo en el Departamento de Ciencias de la Salud ${ }^{(f)}$. En este estudio abordamos el proceso de formación inicial de los estudiantes de la carrera de Medicina desde el comienzo de su organización y puesta en marcha. La intención es comprender en qué consisten los cambios sociales y culturales que se producen en las comunidades, en las instituciones, en los sujetos asociados a la implementación de esta currícula considerada innovada. El encuadre metodológico es de tipo cualitativo-etnográfico. La línea de este trabajo enfoca el relato y la reflexión en torno a cómo se aprende en el escenario "Campo", en los encuentros entre los estudiantes y los equipos de salud, entre los estudiantes y los docentes, entre los estudiantes y la comunidad y entre sí particularmente durante el "práctico reflexivo".

El Municipio de La Matanza se encuentra ubicado en la provincia de Buenos Aires, dentro del Gran Buenos Aires. Es el municipio más extenso del conurbano, con una superficie total de 325,71 kilómetros cuadrados ${ }^{(g)}$. Se caracteriza por su población numerosa, que asciende a 1.775.816 habitantes según el Censo 2010 realizado por el INDEC(h). En 2001 (INDEC) la población que habitaba en hogares con necesidades básicas insatisfechas (NBI) representaba el 20\%. La Universidad de la Matanza comenzó a funcionar en el año 1991 y, de la matrícula total, aproximadamente el $80 \%$ de sus estudiantes que son primera generación de universitarios. En este contexto se enmarca la creación de la carrera de Medicina que comienza a dictarse en el año 2012, como parte de la creación del Departamento de Ciencias de Salud, en 2011, con un proyecto pedagógicodidáctico innovador tanto en cuanto a las decisiones sobre la perspectiva de salud con fuerte enfoque en APS, como por el modo en que están estructurados los espacios curriculares, en términos de espacio-tiempo, centralidad en el aprendizaje, y la producción de conocimiento para la educación médica.

(a-e) Carrera de Medicina, Departamento de Ciencias de la Salud, Universidad Nacional de la Matanza. Florencio Varela 1903 (B1754JEC),

San Justo. Buenos Aires, Argentina. investigacionessalud@ unlam.edu.ar

\footnotetext{
(f) Se trata del proyecto de investigación "La educación médica innovada; cotidianeidad debates y experiencias educacionales en la implementación de una nueva carrera".

(g) Municipio de La Matanza. http://www. lamatanza.gov.ar/ matanza/ubicacion.php

(h) Cuadro P2-D. Provincia de Buenos Aires, partido La Matanza. Población total por sexo e índice de masculinidad, según edad en años simples y grupos quinquenales de edad. Año 2010. http://www. censo2010. indec.gov.ar/ resultadosdefinitivos.asp
} 
Esta carrera de medicina se aprueba e implementa sobre la base de una currícula innovada de educación médica. Los rasgos de esta condición de innovada consisten en que su diseño se centra en el estudiante, se promueven múltiples formas de integración, se prioriza la vinculación teoría práctica y en que los estudiantes realizan experiencias preprofesionales desde el inicio de la carrera. De ahí que los contenidos, prácticas y estrategias pedagógicas se organizan sobre el supuesto del aprendizaje situado y significativo. Es decir, no como la simple aplicación de conceptos y herramientas "neutras" de enseñanza preestablecidas, sino como un ejercicio constante de aprehensión de la realidad considerando al conocimiento como resultado de la actividad de los sujetos, el contexto y la cultura. En cuanto a la relación con el conocimiento, se promueve la autonomía del estudiante para que realice sus propias búsquedas de información con criterio crítico, organice experiencias significativas y construya colectivamente el saber, poniendo a disposición herramientas para la selección de conocimientos válidos y atendiendo los contextos de producción.

Todas las carreras de medicina tienen prácticas preprofesionales, pero en la gran mayoría de ellas estas prácticas son al final de la carrera de grado. La enseñanza de la medicina en la Argentina incorporó en la década del 60 del siglo pasado como resultado de una lucha estudiantil, la práctica preprofesional en hospitales para los estudiantes de la UBA a partir del ciclo clínico, bajo el nombre de unidad hospitalaria (UDH). Esta experiencia, innovadora en su momento, podríamos compararla con la innovación de la carrera de medicina de la UNLaM, en tanto presenta una práctica preprofesional desde el inicio del primer año, que se mantiene y amplía hasta el final de la carrera. En la Argentina, sobre trece carreras de medicina que se dictan en universidades públicas, actualmente ocho implementan currículas innovadoras. Sin embargo, ninguna de las ocho ha incorporado un escenario de aprendizaje concreto y sostenido durante toda la carrera de práctica preprofesional en terreno. La particularidad de la carrera de medicina de la Universidad de la Matanza es que los estudiantes las realizan desde el primer año de la carrera; el escenario curricular en el que las realizan lo denominamos "campo". Llamamos escenario a espacios-tiempo curriculares regulados de forma distinta a los de las "clases convencionales". Son espacios de práctica integradora de saberes provenientes de la propia experiencia y de los aprendizajes que logran en otros escenarios y unidades de aprendizaje, en el que la experiencia es la articuladora. Al espacio "campo" lo constituyen los centros de salud y los ambientes comunitarios y locales en los que estos centros están contextualizados. Durante el año 2012 las instituciones de desempeño fueron centros de salud del municipio de La Matanza, donde está ubicada la Universidad, y de zonas aledañas a este, Morón y zona oeste de la Ciudad Autónoma de Buenos Aires. Se conformaron 14 comisiones de entre 7 y 10 estudiantes cada una, coordinada por un docente tutor de la Universidad que a su vez, en todos los casos, era profesional integrante del equipo de salud del centro de salud base de la comisión - médico/as, enfermera, psicólogas, trabajadoras sociales, bioquímico, odontóloga. En este sentido, podemos afirmar junto a Tejada Fernández que "el profesor no puede seguir siendo el monopolizador del saber y transmisor de conocimiento (modelo tradicional), sino que cobra nuevo protagonismo, en cuanto a competencias sobre todo, por la interacción entre las instituciones educativas y formativas, por un lado, y sociedad en su conjunto, por otro"1 (p. 113). Es desde esta perspectiva que se implementó el escenario "campo", para que los estudiantes estén en contacto, desde el inicio de su carrera, con otras profesiones diferentes a las de su elección del equipo de salud y reflexionen sobre la importancia y la complejidad del trabajo en equipo.

El escenario tiene una carga horaria semanal de cinco horas. Durante las três primeras horas los estudiantes desarrollan en el terreno las actividades programadas; al finalizar, junto con el/la tutor/a desarrollan un plenario y cierran con una sesión de práctica reflexiva. Las prácticas reflexivas tienen como objetivo recuperar saberes y concepciones presentes en las prácticas con el fin de comprender la lógica que las organiza y analizar críticamente las propias prácticas, las de los integrantes de los equipos de salud y las de la comunidad con la que interactúan.

El escenario "campo" es entonces un espacio en el que los estudiantes se contactan con la realidad de las comunidades y con su situación de salud. En este escenario se ponen en juego y se integran necesariamente los conocimientos que se elaboran en las distintas unidades de aprendizaje de la carrera. Asimismo, la presencia de los estudiantes en las instituciones barriales y municipales genera situaciones nunca antes experimentadas que interpelan, incomodan y obligan a la reflexión sobre el 
(i) Manual de escenario "campo".

(j) Estos roles se corresponden con las dimensiones de la Atención Primaria de la Salud. Se apunta a que la formación básica del estudiante de medicina abarque el conjunto de estas dimensiones como manera de evitar reducir la práctica del médico a una función restringida. rol y las competencias de la Universidad y específicamente de los estudiantes de medicina, en su interacción con el medio. Se desdibuja la clásica idea de "adentro y afuera" de la Universidad, cuyos límites comienzan a permearse y a volverse imprecisos.

En "campo" los estudiantes exploran aspectos asistenciales, incorporan habilidades clínicas, aprenden a "ver" y "escuchar" desde el lugar que ocupa la comunidad y los trabajadores de la salud y a documentar lo observado, participan e intercambian con gente de las diferentes comunidades, identifican necesidades, priorizan problemas, formulan planes educativos, preventivos y de promoción ${ }^{2}$.

La programación del escenario para los estudiantes de primer año tiene por objetivo que los estudiantes observen y comprendan el proceso de saludenfermedad - atención desde el punto de vista de la estrategia de la promoción - prevención en APS en Centros de salud y otros espacios e instituciones situadas en el territorio asignado, a través de actividades que los involucren ${ }^{(i)}$.

El programa del escenario se desarrolla en tres etapas. La primera se desarrolla durante cuatro semanas a través de encuentros en el edificio de la universidad. Se considera que deben prepararse para comenzar las actividades en el territorio que les es asignado. Desarrollan actividades de observación participante, entrevistas y registro para ejercitarse en el uso de estos modos de aprehender fenómenos sociales. También toman contacto con los tutores y a través de ellos con primeras informaciones con el lugar y el centro de salud para poder insertarse desde su rol de estudiantes. Las siguientes dos etapas se desarrollan en los centros de salud, instituciones, viviendas y también en la calle en interacción con los trabajadores de los equipos de salud, pacientes, vecinos, docentes, etc. Durante esta segunda etapa los estudiantes comienzan a familiarizarse con las actividades, los modos de actuar y decir, las formas de relación propias de lo local - del Centro de salud, el equipo, los grupos sociales que integran la comunidad de ese barrio y otras instituciones barriales. En la tercera etapa se continúan realizando las actividades de la etapa previa pero se espera que se espera que los estudiantes vayan incorporando y afianzándose en los diferentes roles que deben asumir como parte fundamental del aprendizaje en este escenario: promotor y defensor de derechos, educador, agente sanitario, investigador, preclínico ${ }^{(j)}$. Durante esta última etapa, cada comisión implementa un proyecto de investigación participativa que se va construyendo a partir de la detección de necesidades y del intercambio con la comunidad y el equipo de salud. Se trata de que los estudiantes aprendan a formular preguntas que permitan problematizar las situaciones de conflicto, demanda, necesidad como modo de comprenderlas, y diseñar posibles intervenciones que intenten dar respuestas acotadas a las situaciones problematizadas. Entre las situaciones que han sido problematizadas por los estudiantes de primer año podemos mencionar caídas de altura por déficits constructivos en las viviendas, primeros auxilios ante quemaduras y mordeduras de animales, sexualidad en adolescentes, la convivencia con la basura.

Así, durante las tres etapas, los estudiantes generan situaciones en las que se encuentran con grupos y personas con quienes interactúan y despliegan formas dialógicas que les abren las posibilidades de conocer desde y con la experiencia. De una manera similar al trabajo de campo de los etnógrafos, se promueven encuentros con "otros" con quienes interactúan y construyen experiencias que generan conocimiento en tanto se estimula la circulación de información que deviene en aprendizaje para todos los involucrados. Fabian ${ }^{3}$ sostiene que la etnografía - modo de producir conocimiento antropológico - es dialógica, producto de la interacción fundamentalmente - aunque no solamente - hablada, y enfatiza que los datos que se desprenden de la investigaciones se forjan, se 
hacen; no se encuentran - como se considera habitualmente. Quienes se encuentran son personas, sujetos que se comunican y a través de los lenguajes y en la acción producen colaborativamente conocimiento. Tomamos esta conceptualización de "encuentro" para hacer una lectura comprensiva del escenario y preguntarnos en qué consisten los encuentros en los que se aprende y se enseña con la presencia de otros que no son ni estudiantes ni docentes.

\section{Encuentros en el escenario campo ¿Quiénes son los que se encuentran en "campo"?}

Nos preguntamos en un primer momento quienes son lo que se encuentran; pensamos que en primer lugar lo hacen los estudiantes entre sí; progresar desde el encuentro de individualidades hacia la conformación de los grupos en términos psico-sociales y la integración como equipos de trabajo.

Concomitantemente al encuentro entre los estudiantes, se produce el encuentro entre estos y sus tutores $y$, posteriormente, el encuentro entre los estudiantes y los equipos de la salud y entre los estudiantes y la comunidad. Si bien ordenamos los encuentros secuencialmente, todos estos relacionamientos devienen dialécticamente durante toda la experiencia de "campo" e impactan en los sujetos, grupos, instituciones y comunidad, pero particularmente en los procesos y experiencias de formación de los estudiantes. Esto produce que cada estudiante esté atravesado y en continua modificación por todas estas vivencias. Lo que sucederá también con cada uno de los protagonistas de estos colectivos, es decir, los tutores, los integrantes de los equipos de salud, los pobladores de esa localidad/barrio/vecindad.

Dándonos una licencia para generalizar, podríamos afirmar que en los primeros momentos de la llegada de los estudiantes a los centros de salud, la representación de los profesionales de los equipos acerca de ellos, consistió en pensar que eran demasiado jóvenes y que aprenderían como "tablas rasas" o recipientes vacíos sin ningún conocimiento previo, acerca de la realidad que abordarían; o, en el mejor de los casos, tenían una incipiente y por lo tanto muy escasa capacitación teórica lo que indicaba que no podrían integrarla a las experiencias en el terreno. Pero, la lógica integradora como rasgo que atraviesa toda la currícula - tanto las unidades de aprendizaje, los otros escenarios - y la preparación previa en el escenario "campo" abrían otras oportunidades que muy pronto se impusieron sobre esa representación descripta. Las prácticas pre profesionales se fueron constituyendo en experiencias motivadoras y potenciadoras de las saberes abordados en otros espacios, actualizaron experiencias culturales de los estudiantes, promovieron el estudio y le dieron sentido a muchos de los contenidos abordados en la enseñanza-aprendizaje, por ejemplo en los espacios de seminario, de clase en la universidad. Entender cómo los saberes teóricos organizan y dan sentido a las prácticas, es decir reconstruir supuestos teóricos organizadores de las prácticas era una tarea central en campo; y en correspondencia con esto era muy importante que los estudiantes estuvieran atentos a descubrir cuándo y cómo las prácticas interpelaban, se relacionaban, se correspondían, se apartaban o acercaban a conocimientos enseñados teóricamente en las unidades de aprendizaje. Para que esto fuera posible se ofrecían oportunidades para vivir experiencias e incorporar herramientas de aprendizaje que permitían que tanto estudiantes como tutores desarrollaran procesos de distanciamiento y aproximación a lo vivido. Así se intentaba que tendieran puentes cognitivos y emocionales entre las vivencias, las significaciones y los saberes habitualmente incluidos entre los teóricos para integrar, resignificar y producir conocimiento. Una herramienta valiosa para el registro y análisis de las experiencias es el cuaderno de campo en donde los estudiantes aprenden a anotar en forma de relato, descripción o simple anotación lo que observan, escuchan, hacen, dicen, sienten. Los estudiantes desarrollan muchas actividades de observación participante que incluye las conversaciones y, al registrar lo que alcanzan de estas vivencias, aprenden a observar y escuchar, a estar con los otros. En los cuadernos de campo objetivan a través de la escritura lo vivido. Esto les permite leer y analizar lo pensado y sentido durante los encuentros y así ir desarrollando una visión crítica-vivencial de la 
(k) Utilizamos el término in-corporar para enfatizar la idea que esta capacidad comunicacional se encarna, se hace cuerpo en los estudiantes.

(l) El Dr. Mario Rovere denomina nivel 0 del sistema de salud a aquel que el sistema NO incluye en su organización, que algunos profesionales intentan abordar y que nuestro programa focaliza como el nivel que más oportunidades brinda para

desarrollar creatividad en estrategias de APS, que fomenta la equidad en las posibilidades de acceso y que ofrece también las mejores oportunidades para que los estudiantes entren en contacto directo con modo no biomédicos de atención a la salud. realidad y una capacidad comunicacional que los pone en escena como integrantes del equipo de salud y les permite in-corporar ${ }^{(k)}$ dicha capacidad para utilizarla en el futuro quehacer profesional.

Los estudiantes son modificados por lo que van vivenciando día a día; del mismo modo, los profesionales de los equipos de salud - entre ellos los propios tutores/as - comienzan a ser modificados por la interacción con los estudiantes. Con esta afirmación, queremos subrayar que el hecho que los estudiantes asistan a desarrollar actividades en terreno, por un lado, impacta en los procesos de aprendizaje de los propios estudiantes en dimensiones experienciales diferentes a los modos de aprender en seminarios, laboratorios, talleres, tutorías, ateneos, etc. Por otro lado, la presencia de estudiantes desde el primer año en los lugares donde trabajan los técnicos y profesionales de los equipos de salud, provoca un impacto altamente positivo en varias dimensiones. Entre ellas observamos que los trabajadores de la salud se implican en actividades docentes y agradecen esta oportunidad ya que, al exponerse frente a los estudiantes, mejoran la calidad de la atención brindada y se sienten estimulados a actualizar sus conocimientos, tal como varios tutores lo han expresado. En cuanto a los tutores, adquieren habilidades de coordinación de grupo no sólo porque tienen que organizar y dinamizar las comisiones de estudiantes, sino también, porque la propia tarea de coordinación tutorial requiere el armado de redes tanto con el equipo de salud - dentro del centro de salud -, como con otras instituciones - escuelas, comedores, centros de jubilados - y con hospitales. Esto produce modificaciones en los vínculos de ese profesional que es tutor y de los referidos a los centros de salud que densifican sus redes, tanto institucionales como personales. Estas situaciones han redundado en beneficios para los usuarios.

La noción que subyace a la observación participante es que para aprender es necesario desarrollar la capacidad perceptiva - en este caso sobre todo la observación y la escucha - y de interacción con personas y grupos a través de la participación que permite insertarse en los modos de hacer, pensar y actuar. A la vez, este aprendizaje requiere cierto grado de separación de la realidad tal como es vivida para alcanzar grados de comprensión cada vez más profundos. De ahí que, como parte de las relaciones que se van construyendo en los centros de salud y otros lugares de la localidad, los estudiantes desarrollan un proceso de proximidad con pobladores y trabajadores de la comunidad local en el llamado nivel 0 del Sistema de Salud ${ }^{(1)}$ que produce lo que hemos denominado provisoriamente "proceso de cercanía". Se conforma una suerte de alianza entre actores de ambos grupos, de confianza mutua y colaboración, en la cual los estudiantes se valorizan y son valorizados, y los integrantes de la comunidad local se sienten escuchados y atendidos en forma diferente, humanizada. A punto tal que algunos de los vecinos se llegan a considerar de manera explícita co-formadores de sus futuros médicos. La población local vislumbra el lugar que ocuparán esos estudiantes en un futuro, puede darse el permiso de aconsejarlos, de explicitar lo que ellos esperan de un médico, de hacer y decir "cosas" que no se atreverían a comunicarle a un profesional de la salud. Los estudiantes en un comienzo se posicionan con cierta distancia y ajenidad, no sabiendo como ocupar ese lugar de médicos que no los implica aun, pero escuchan y reciben. Ellos están en una posición privilegiada para interpretar las demandas y deseos de los vecinos y pobladores del lugar a los que algún día van a atender como médicos. Este "proceso de cercanía" abre mejores posibilidades para formarse como médicos incorporando desde el inicio en 
forma combinada y a veces poco discernible, impresiones, emociones y conocimientos que marcarán de una manera particular el modo de hacer y pensar la salud, un modo particular de entender y entender-se con los otros. Los auténticos encuentros transforman a los sujetos y su realidad, aun en los desacuerdos.

En palabras de uno de los coordinadores del escenario, el objetivo a lograr es que los estudiantes se sientan "como pez en el agua en la comunidad". Cooke et al. ${ }^{4}$ refieren que la formación profesional ocurre a través de la relación con los pacientes. Los autores manifiestan que dicha relación involucra, desde los primeros momentos de la carrera hasta la práctica profesional, experiencias que quedan fijadas, con emociones intensas o resolución de puntos de vista conflictivos o dilemas éticos. Postulan que estas experiencias forman los valores personales y el compromiso con el fin de sembrar el deseo de abogar en defensa de los mejores intereses del paciente.

Los autores referencian a Bleakley y Bligh ${ }^{5}$ quienes fueron aun más lejos asegurando que las relaciones profundas, de colaboración, entre los estudiantes y los pacientes deben ser consideradas como el lugar principal para la producción de conocimiento, ya que esta relación es la que motiva que el estudiante pueda comprender la condición de ese paciente tanto desde la perspectiva del paciente, como desde una perspectiva biomédica y psicológica, a la que nosotros agregamos las dimensiones social y cultural. Es más, retomando lo ya enunciado, esta motivación impulsa también a investigar y a reflexionar sobre la práctica.

\section{¿Qué lugar ocupa lo incierto?}

Para conocer y entender es preciso ser activo y desarrollar autonomía. "Nadie aprende el oficio de connoisseur o el de diagnosticador si se limita a poner en práctica reglas preexistentes. En este sentido entran en juego (se dice habitualmente) elementos imponderables; olfato, golpe de vista,

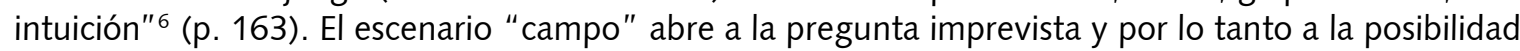
de aprender de la perplejidad, de la sorpresa, del desconcierto, del asombro, de la extrañeza y así develar y descubrir, según los casos. Develar y descubrir produce aprendizajes que están vinculados a conocimientos previamente incorporados. De esta manera se promueve la curiosidad del estudiante, situándolo en otra práctica explícitamente formulada como parte de su formación: la de investigador.

En este sentido, Freeth escribe que las teorías sobre aprendizaje resaltan la importancia motivacional del "gap" entre lo que la gente cree saber y lo que la gente piensa que necesita saber?. La autora refiere que esta brecha, estimula el aprendizaje y que los educadores ágiles de todos los tiempos han encontrado maneras de crear o revelar disyunturas constructivas. Aquello que los estudiantes descubren y develan en "campo" puede ser analizado de esta manera, lo que explica su valor educacional.

En "Campo", no todo está pautado o estructurado. Queda lugar para la incertidumbre que motoriza el aprendizaje individual y colectivo y la reflexión, como práctica reflexiva y como autorreflexión.

\section{La práctica reflexiva}

La reflexión es clave en el aprendizaje, en la práctica, en tanto y en cuanto es lo que une "el aprender" con "el hacer" 8 . Ahora bien, es necesario que ese aprendizaje en el caso de un estudiante de medicina sea guiado. Es por esto que la práctica reflexiva se realiza junto a los tutores y coordinadores del escenario. Los espacios destinados a la práctica reflexiva apuntan a consolidar una formación informada tanto por la práctica como por la teoría. Es decir, a comprender que el conocimiento no está reducido a la teoría sino que también está en la acción otorgando una lógica a las prácticas, muchas veces difícil de explicitar y, por lo tanto, analizar. La práctica reflexiva tiende a evitar el riesgo de autorizar a la práctica por sobre los conocimientos académicos, como a éstos por sobre la práctica. Así conocimientos académicos y prácticos pueden encontrar modos de articulación e integración apropiados. Los espacios destinados a realizar prácticas reflexivas son privilegiados en tanto dan la oportunidad para la articulación concreta de conocimientos en un sentido transductivo. 
El concepto de práctica reflexiva vinculado al aprendizaje fue utilizado por John Dewey en un libro denominado "Cómo pensamos" 9 , para referirse a que la actividad de los seres humanos se sostiene en intuiciones y en saberes previos, y por lo tanto cualquier problema lo abordamos, en principio, apelando a ciertos modos de comprensión habituales que hemos adquirido. Esto significa que siempre reflexionamos desde lo que sabemos y hemos vivido, y lo vivido para ser comprendido requiere ser organizado. Es precisamente hacia la necesaria organización de las experiencias vividas, que apuntan los momentos curricularmente programados para realizar las prácticas reflexivas, las que podemos definir siguiendo a Schön ${ }^{10}$, como instancias grupales para el proceso de examinación y exploración interna de un tema de preocupación, provocado por una experiencia, que crea y clarifica significados en términos de sí mismo, y que se traduce en una perspectiva conceptual modificada.

Por otro lado, debe enunciarse que la reflexión puede realizarse en el momento mismo de la práctica o después de esta. Este último caso es al que apunta la propuesta de la práctica reflexiva, que se realiza al finalizar cada jornada semanal en campo. El lugar físico en donde se realiza alterna quincenalmente. Una semana es en el edificio de la Universidad donde se encuentran estudiantes que desarrollan actividades en distintos centros de salud y territorios - alrededor de 40. La semana siguiente es in situ, en el centro de salud donde trabaja cada grupo, y la práctica reflexiva se realiza entre los integrantes de ese grupo - 10 estudiantes.

La práctica reflexiva tiene por objetivo inmediato desarrollar la capacidad de reflexionar sobre lo actuado, vivenciado, para entender los supuestos que dieron sentido a las interacciones, las acciones, las experiencias. A través de estas reflexiones se procura que las prácticas adquieran el estatus de conocimiento y permitan así ser interrogadas, interpretadas y corregidas. Por otro lado, la práctica reflexiva puede entenderse como un entrenamiento para que el futuro profesional adquiera los hábitos de la formación permanente, tan necesaria para un profesional de la salud.

Esta metodología de acompañar las experiencias preprofesionales tempranas en la formación de médicos con prácticas reflexivas y como parte del aprendizaje de la estrategia de la Atención Primaria de Salud en el marco de la comprensión integral del proceso salud-enfermedad-atención es indudablemente una innovación en el campo de la educación médica.

\title{
El escenario en relatos de sus protagonistas
}

"Fue impactante porque pude darme cuenta de cosas que suceden cerca del lugar en el que vivo y que antes de la visita a ese lugar no sabía que pasaban. Siempre viví en Laferrere y hasta ese día nunca supe que hay barrios en los que cuando llueve se inundan y se destruyen las casas de muchos vecinos, teniendo éstos que ser evacuados. Me impactó tanto el relato de estas personas que después busqué en internet noticias sobre inundaciones y pude ver que esto es un tema grave". (Estudiante, cuaderno de campo, abril 2012)

\begin{abstract}
"A mi me tocó ir con una compañera a conversar con dos familias, y las historias de ambas eran similares, vivían en condiciones de vida muy humildes y luego de la inundación se habían quedado en la calle con todos sus hijos, no tenían dinero y casi ninguna pertenencia pero nos trataron tan bien... Fueron muy atentas con nosotras, la charla fue muy fluida. Por otra parte me sentí un poco indignada con algunas cosas que nos contaron las mamás de los niños, con respecto al trato que recibían en la escuela que los acogía. Decían que no les daban de comer bien y que las cosas que la gente donaba para ellos no se las daban sino que se las quedaban o las utilizaban delante de ellos. Al ver estas cosas uno puede intentar ponerse en el lugar del otro y darse cuenta de que lado quiere estar, si ayudando a quienes necesitan o abusando y aprovechándose de esa necesidad. A mi me encantaría poder formar parte de los primeros". (Estudiante en relación a visita a un centro de evacuados, mayo 2012)
\end{abstract}

Durante una práctica reflexiva: Los estudiantes de la comisión 6 dicen que en el día de hoy estuvieron hablando con una referente del barrio para hacer el relevamiento del hábitat. Mencionan 
que esta señora vive a sólo unas cuadras del centro de salud pero que las condiciones de vida cambian abruptamente. Que no tiene luz ni agua potable. Están contentos con este encuentro, dicen que no querían "ir a ver lo que el mundo o todos quieren escuchar". Están muy impactados por el encuentro. Cuentan cómo vive la señora, dicen que les hizo recorrer su casa, que tiene un chancho y otros animales. Entre todos cuentan el encuentro. "Yo en mi propio barrio no conocía esa realidad". "Para mi es más importante que ellos vean que nos importan, no sólo darles salud, salud, salud". "Ellos están acostumbrados a que ellos vienen a nosotros" (Práctica reflexiva 27-9-2012, estudiantes comisión 6).

\section{Conclusión}

En base a lo ya recorrido podemos afirmar que las prácticas pre profesionales tempranas son beneficiosas e indispensables para la formación de profesionales idóneos y comprometidos con la realidad de la población a la que van a atender. En este sentido, a diferencia con las formaciones de médicos que se incorporan a prácticas preprofesionales recién en la fase clínica, esta forma temprana permite incorporar - en el sentido de hacer cuerpo (embodiment) - desde la experiencia una visión amplia del rol del médico en la APS, produciendo que no reduzca su práctica a cuestiones de índole clínico asistencial, sino que éstas se integren a roles tales como los de educador, defensor de derechos, investigador, entre otros. Consideramos que esto le permitirá reconocer, por ejemplo, aspectos indispensables de las estrategias de APS como la interculturalidad, no en el sentido de un discurso, sino de una práctica en sí misma, de un modo de establecer relaciones y comunicación con los otros.

Es asi que, en base a nuestra experiencia, consideramos fundamental que el estudiante participe de la resolución de problemas complejos de salud. Refiere Marins ${ }^{11}$ que de esa forma los estudiantes pasan a participar de las reuniones de las asociaciones de vecinos, de los consejos de salud y de otros espacios donde esos problemas son debatidos. Esta primera investigación nos lleva a coincidir con la postura del autor que considera que "las habilidades y actitudes que el estudiante puede desarrollar en su actuación frente a los problemas concretos de la realidad, ejercen una enorme influencia sobre la postura de los futuros profesionales"11 (p. 100). Marins propone, al igual que nosotros, que los estudiantes comiencen la experiencia de campo en el primer año de la carrera de medicina.

En esta primera exploración fuimos encontrando algunos términos ya utilizados por otros autores ${ }^{12}$. Entre los más importantes señalamos que los "descubrimientos" en el "contacto con situaciones reales", son relevantes a los fines del aprendizaje significativo y de la mirada social que se espera que tengan los estudiantes de nuestras carreras. Por otra parte, desde nuestra propia experiencia, estos "descubrimientos" pueden producirse desde más temprano en la carrera, dejando entonces huellas más profundas que van a incidir en el futuro quehacer profesional.

A esto se suma el hecho que desde la organización del escenario campo apuntamos a que los estudiantes vayan en el primer año a campo en sus zonas de residencia. En este sentido, otra vivencia que hará mella en los futuros profesionales es la de "descubrir" en sus propios barrios, en sus lugares de residencia, situaciones de vida similares y diferentes a las suyas y así alcanzar grados de alteridad imprescindibles para la práctica médica.

Destacamos el lugar privilegiado de la práctica reflexiva que permite que lo vivido en los encuentros en campo puedan pensarse y reconstituirse como conocimientos situados. Como fue enunciado en este trabajo, el escenario campo no podría darse sin las instancias de reflexión. Nos referimos a la reflexión tanto durante la acción, y para el caso particular del estudiante de medicina es la acción propia y la acción observada en el escenario. Nuevamente, se trata de una reflexión que surge de la sorpresa ante lo inesperado ${ }^{10}$.

Pero a los fines de la enseñanza de la medicina, es necesario e imprescindible que esas reflexiones durante la acción, sean luego revisadas y encauzadas a un aprendizaje significativo. Dado que las experiencias prácticas están en el centro de la formación profesional del médico, las prácticas reflexivas estimulan y habilitan los procesos de transformación de experiencias en conocimientos, habilidades, actitudes y valores. Durante las prácticas reflexivas los estudiantes, junto con tutores y profesores, se preguntan: sobre problemas acontecidos en las actividades de Campo, sobre sus consecuencias y cómo 
fueron abordados, sobre lo que se aprendió del problema y del sí mismo, en esa situación y sobre cómo una situación igual o similar podría ser abordada la próxima vez. $Y$, muy en particular, sobre elementos del bagaje teórico que van incorporando en las distintas unidades de aprendizaje y "cobran vida" en las experiencias que se producen en campo. Allí, la producción de conocimiento alcanza una dinámica particular y situada: es donde estamos, con quienes estamos y en nosotros mismos. Esta metodología de trabajo puede constituir otra forma de "ser médico" que facilite las miradas integrales e integradoras de las prácticas en salud.

Quedan por delante muchos desafíos:

- Consolidar la articulación del escenario "campo" con el resto de la currícula.

- Co-construir la articulación con los servicios de salud.

- Lograr llegar a ser agentes transformadores del Sistema de Salud mediante el protagonismo de los estudiantes en sus procesos de aprendizaje en el escenario.

- Superar las dificultades que surgen del conflicto de lealtades de los tutores de campo hacia sus centros de salud y hacia la universidad.

- Vencer nuestra propia tendencia a reproducir las formas en las que aprendimos, "no enseñar cómo nos enseñaron".

\section{Colaboradores}

Los autores trabajaron juntos en todas las etapas de producción del manuscrito.

\section{Referencias}

1. Tejada Fernández J. Función docente y formación para la innovación. Rev Acad Mex Educ. 2001; 4(4):111-38.

2. Milstein D, Mazur V. "Campo" y práctica reflexiva. Texto escrito para estudiantes de la carrera de Medicina de la UNLaM (inédito). San Justo; 2013.

3. Fabian J. Time and the other: how anthropology makes its object. New York: Columbia University Press; 1983/2002.

4. Cooke M, Irby D, O'Brien B. Educating physicians: a call for reform of medical school and residency. San Francisco: Jossey-Bass; 2010.

5. Bleakley A, Bligh J. Who can resist Foucault? J Med Philos. 2009; 34(4): 368-83.

6. Ginzburg C. Mitos, emblemas, indicios: morfología e historia. Barcelona: Gedisa; 2010.

7. Freeth D. Interprofessional education. En: Swanwick T, editor. Understanding medical education: evidence, theory and practice. Oxford: Wiley-Blackwell; 2010. p. 53-68.

8. Pee B, Woodman T, Fry H, Davenport ES. Practice-based learning: views on the development of a reflective learning tool. Med Educ. 2000; 34(9):754-61.

9. Dewey J. Cómo pensamos. Nueva exposición de la relación entre pensamiento reflexivo y proceso educativo. Barcelona: Paidós; 1933/1989.

10. Schön D. La formación de profesionales reflexivos: hacia un nuevo diseño de la enseñanza y el aprendizaje de las profesiones. Barcelona: Paidós; 1987.

11. Marins NJJ. Los escenarios de aprendizaje y el proceso del cuidado de la salud. En: Marins NJJ, Rego S, Barbosa Lampert J, Correa de Araújo JG, organizadores. Educación médica en transformación: instrumentos para la construcción de nuevas realidades. São Paulo: Hucitec-ABEM; 2004. p. 98-102.

12. Arango de Bedoya Y, Casas LA, Grill H, Hoyos J, Jimenez F, Montoya JG, et al. Práctica comunitaria en unidades de atención primaria a nivel rural: una experiencia con estudiantes de medicina. Educ Med Salud. 1984; 18(2):164-84. 
En el año 2012 el Departamento de Ciencias de la Salud de la Universidad de la Matanza, Argentina, implementó la carrera de medicina. Sobre la base de un diseño curricular que integra conocimientos y prácticas, los estudiantes, desde el primer año de la carrera, desarrollan actividades en la comunidad junto a equipos de salud. Con este fin se generó un escenario de aprendizaje denominado "Campo". Este trabajo describe la experiencia del primer año de la primera cohorte de estudiantes de la carrera, específicamente en relación a la inserción en este escenario, y discute alrededor de este modo de enseñanza y aprendizaje innovador, que consiste en incorporar a los estudiantes a observar y participar junto a los equipos de salud de los centros del primer nivel de atención.

Palabras clave: Educación médica. Innovación. Prácticas pre-profesionales. Práctica reflexiva.

\section{"Field" in medical education: a scenario for putting healthcare and education practices into perspective}

In 2012, the Department of Health Sciences of the University of La Matanza, Argentina, implemented an undergraduate medical course. Based on a curriculum that integrates knowledge and practice, first-year students on the course develop activities with healthcare teams in the community. For this purpose, a learning scenario called "Field" was generated. This paper describes the experience of the first year of the first group of students, specifically in relation to their inclusion in this scenario, and discusses this innovative teaching and learning process, in which students observe and participate in health teams at primary care units.

Keywords: Medical education. Innovation. Pre-professional practices. Reflective practice.

\section{"Campo" na educação médica: um cenário para colocar em perspectiva práticas de Saúde e Educação}

Em 2012, o Departamento de Ciências da Saúde da Universidade de La Matanza, Argentina, implementa o curso de graduação em Medicina. Com base em um currículo que integra conhecimento e prática, os alunos do primeiro ano do curso fazem atividades com as equipes de saúde na comunidade. Para este fim, foi gerado um cenário de aprendizagem denominado "Campo". Este artigo descreve a experiência do primeiro ano do primeiro grupo de estudantes, especificamente em relação à inclusão nesse cenário, e discute sobre o processo inovador de ensino e aprendizagem em que alunos observam e participam, com as equipes de saúde, das unidades de atenção primária.

Palavras-chave: Educação médica. Inovação. Prática pré-profissional. Prática reflexiva. 